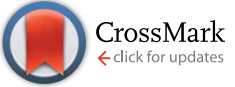

Cite this: RSC Adv., 2016, 6, 113689

Received 3rd September 2016 Accepted 28th November 2016

DOI: $10.1039 / c 6 r a 22084 c$

www.rsc.org/advances

\section{Profiling changes to natively-bound metals during Caenorhabditis elegans development $\uparrow$}

\begin{abstract}
Dominic J. Hare, $t^{\mathrm{ab}}$ Blaine R. Robertst $t^{\mathrm{b}}$ and Gawain McColl ${ }^{\mathrm{b}}$
Relatively little is known about the changing metalloproteome during early development. In this proof-ofconcept study, we used size exclusion chromatography-inductively coupled plasma-mass spectrometry (SEC-ICP-MS) to examine the changing soluble metal-binding protein profiles for iron, copper and zinc during the development of the nematode, Caenorhabditis elegans. Samples of eggs, larval stages and young adults were compared using an approach selected to ensure weak metal-ligand bonds were maintained. All three metals showed marked changes in associated proteins and total metal levels per protein mass, and the pattern of this change was unique to each metal. Additionally, to characterise the shifting metabolic needs throughout each life stage we examined changing levels of phosphorus in each developmental stage. The utility of this method can be further exploited through integration with existing proteomics workflows to identify and track the changes in metal-containing proteins during key stages of development.
\end{abstract}

\section{Introduction}

The direct examination of metalloproteins from biological samples is an evolving analytical approach. ${ }^{1}$ Although specific metalloenzymes have been the focus of investigation, this has largely been through a piecemeal process with the majority remaining uncharacterised. ${ }^{2}$ It is estimated that at least one-third of the human proteome interacts in some form with a metal ion, ${ }^{1}$ through either functional or structural conformations.

The nematode Caenorhabditis elegans is an established animal model of early development, and has more recently been shown to be amenable for studying biologically relevant metals in a complex biological system..$^{3-5}$ Approximately $38 \%$ of the predicted protein-encoding genes of the C. elegans genome have a human ortholog. ${ }^{6}$ In combination with the characterised genetics and stereotypic development, C. elegans provides an excellent basis to study how metal ions participate in and regulate a range of biological functions.

As adult hermaphrodites, C. elegans lay self-fertilised eggs that proceed through four larval stages of development (L1 to L4), prior to reaching reproductive maturity. Adult hermaphrodite C. elegans possess a total of 959 somatic cells, ${ }^{7}$ which continue to age without further mitotic events. We have previously examined how metalloprotein function, specifically the homologues of ferritin, becomes dysfunctional with age and is susceptible to

${ }^{a}$ Elemental Bio-imaging Facility, University of Technology Sydney, Broadway, New South Wales, Australia

${ }^{b}$ The Florey Institute of Neuroscience and Mental Health, The University of Melbourne, 30 Royal Parade, Parkville, Victoria, 3052, Australia. E-mail: gmccol@florey.edu.au $\dagger$ Electronic supplementary information (ESI) available: Supplementary figures and table. See DOI: 10.1039/c6ra22084c

\$ These authors contributed equally. environmental influences. ${ }^{8}$ However, comparatively little is known about how endogenous metal and metal-binding protein levels change during the rapid period of growth from egg to adulthood.

The characterisation of metal-binding proteins can be an analytical challenge, as the native physiological conditions that dictate protein-metal coordination states can be easily disrupted. Consequently, the full complement of the metalloproteome (that is, the entire metal and metal-binding protein component of a cell or organism ${ }^{9}$ ) may still remain largely uncharacterised due to limitations in contemporary analytical methods and a predilection for protein mis-metallation during or following sample preparation. ${ }^{2}$

We have applied separation methods using native size exclusion chromatography (SEC) to preserve metal-protein bonds hyphenated to highly sensitive inductively coupled plasma-mass spectrometry (ICP-MS) to perform online detection of the metal status associated with specific molecular weights of biomolecules. ${ }^{10}$ We have previously used this approach to improve the accuracy of transferrin saturation measurements in human serum, ${ }^{11}$ profile the distribution of metalloproteins in cultured neurons and astrocytes ${ }^{12}$ and examine major iron-binding proteins in adult $C$. elegans during normal biological ageing. ${ }^{8}$ In this paper, we have applied this approach to begin to understand metalloprotein speciation in the developing C. elegans nematode. Our data is consistent with early development being punctuated by marked and specific changes in iron, copper and zinc ligands.

\section{Experimental}

\section{Chemicals and standards}

Anhydrous ammonium nitrate $\left(\mathrm{NH}_{4} \mathrm{NO}_{3}\right.$; Sigma Aldrich, Australia) was used as the buffer for all SEC-ICP-MS experiments. 
$\mathrm{NH}_{4} \mathrm{NO}_{3}$ was made to $200 \mathrm{mM}$ in MiliQ water (18.2 M 2 ), $\mathrm{pH}$ adjusted to 7.7-7.8 with ammonium hydroxide $\left(\mathrm{NH}_{4} \mathrm{OH}\right.$; Sigma Aldrich) and filtered prior to use. Superoxide dismutase-1 (copper, zinc; SOD1) and ferritin (iron) standards (Sigma Aldrich) were diluted to $200 \mu \mathrm{g} \mathrm{L}^{-1}$ and $2000 \mu \mathrm{g} \mathrm{L} \mathrm{L}^{-1}$ total metal, respectively, in the $\mathrm{NH}_{4} \mathrm{NO}_{3}$ buffer and the concentration confirmed using solution nebulisation ICP-MS following dilution in $1 \%(\mathrm{v} / \mathrm{v})$ Suprapur® grade nitric acid $\left(\mathrm{HNO}_{3}\right.$; Merck Serono, Australia) in MiliQ water as previously described. ${ }^{12}$

\section{Culturing of C. elegans developmental stages}

The Bristol strain (N2) was used as wild type ${ }^{13}$ and was maintained on a large scale using $8 \mathrm{P}$ media at $20{ }^{\circ} \mathrm{C}$ supplemented with concentrated E. coli (strain OP50). ${ }^{14}$ Eggs were isolated from gravid hermaphrodites every four days using an alkaline hypochlorite treatment. ${ }^{15}$ For subsequent analysis the following developmental stages were collected: eggs, larval stages L1 through L4 and young adults (YA). Eggs were collected immediately following an alkaline hypochlorite treatment, washed four times in excess $18.2 \mathrm{M} \Omega$ water (Milli-Q) and the slurry frozen in liquid $\mathrm{N}_{2}$. The $\mathrm{L} 1$ larvae were hatched at $20{ }^{\circ} \mathrm{C}$ for 12 hours in S-basal ( $0.1 \mathrm{M} \mathrm{NaCl} ; 0.05 \mathrm{M} \mathrm{KHPO}_{4}$ at $\left.\mathrm{pH} 6.0\right)$ and then washed and frozen as described above. The remaining stages were plated on $8 \mathrm{P}$ media with concentrated OP50 and cultured for an additional 16 hours (L2), 28 hours (L3), 36 hours (L4) and 48 hours (YA).

\section{Sample preparation}

C. elegans samples were sonicated (on ice) in a $1: 1$ volume ratio of Tris-buffered saline ( $\mathrm{pH}$ 8.0) with added proteinase inhibitors (EDTA-free; Roche, Australia). Sample homogenisation was confirmed by microscopic inspection. Lysates were then centrifuged for 15 minutes at $175000 \mathrm{~g}$ at $4{ }^{\circ} \mathrm{C}$. The supernatant was removed and total protein concentration in the soluble fraction was determined using a NanoDrop UV spectrophotometer (Thermo Fisher Scientific, Australia) before being transferred to standard chromatography vials with polypropylene inserts (Agilent Technologies, Australia) and kept at $4{ }^{\circ} \mathrm{C}$ on a Peltier cooler for analysis.

\section{Size exclusion chromatography-inductively coupled plasma- mass spectrometry}

All experiments were performed using an Agilent Technologies 1100 Series liquid chromatography system hyphenated to an Agilent Technologies 7700x Series ICP-MS. The column eluent was directly connected to the ICP-MS through a MiraMist concentric nebuliser (Burgener Research, Canada) via polyethyl ether ketone (PEEK) tubing with an I.D. of $0.13 \mathrm{~mm}$. Helium (flow rate $3 \mathrm{~mL} \mathrm{~min}{ }^{-1}$ ) was used as a collision gas to remove potential polyatomic interferences. Separations were performed on an Agilent Technologies BioSEC 5 SEC column $(5 \mu \mathrm{m}$ particle size, $300 \AA$ pore size, I.D. $4.6 \mathrm{~mm}$ ). $200 \mathrm{mM} \mathrm{NH}_{4} \mathrm{NO}_{3}$ was used as the buffer for all separations at a flow rate of $0.4 \mathrm{~mL} \mathrm{~min}^{-1}$. Molecular weight calibration of the column was performed using a mixture of thyroglobulin (I), ferritin, catalase (both iron), SOD1 (copper, zinc) and a rubidium spike to determine

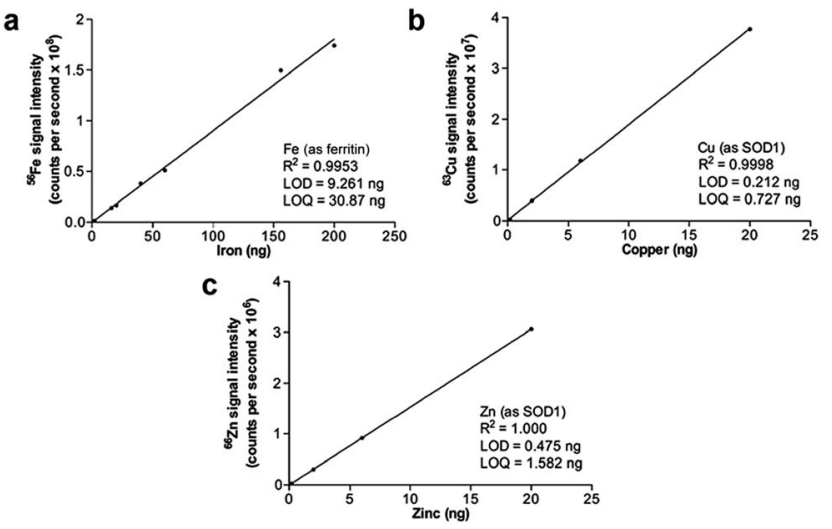

Fig. 1 Calibration curves for (a) iron, (b) copper and (c) zinc, using ferritin ( $\mathrm{Fe}$ ) and superoxide dismutase $1(\mathrm{Cu}$; $\mathrm{Zn}$ ) quantified for metal content by ICP-MS as reference standards.

the permeation limit (ESI Fig. S1 and S2†). Using the protein concentration determined as above, $50 \mu \mathrm{g}$ of total protein was loaded onto the column by manually adjusting the injection volume for each sample. Mass-to-charge ratios $(\mathrm{m} / \mathrm{z})$ for phosphorus (31), iron (56), copper (63) and zinc (66) were monitored in time resolved analysis mode. Solution nebulisation ICP-MS experiments were performed on the same Agilent Technologies 7700x Series ICP-MS.

\section{Data analysis}

Time resolved plots of $\mathrm{m} / \mathrm{z}$ signals were exported from MassHunter (Agilent) and imported into Prism 6.0 h (Graph Pad, USA). All chromatograms were baseline-corrected by subtracting the mean signal intensity of the first 35 recorded data points from each run for each measured $m / z$; and the $x$-axis converted to retention volume $\left(V_{\mathrm{t}}\right.$ in $\left.\mathrm{mL}\right)$. Calibration curves were prepared using increasing injection volumes of prepared standard solutions of ferritin (iron) and SOD1 (copper, zinc) according to metal concentrations predetermined by solution nebulisation ICP-MS analysis (Fig. 1). Total metal levels per $\mu \mathrm{g}$ of protein were determined by integrating the area under the curve and applying appropriate linear regression analysis for respective calibration curves. Limits of detection were determined for each metal according the equation:

$$
\text { LOD } \text { or LOQ }=\frac{x \cdot S_{y}}{m}
$$

where $x=3$ for limit of detection (LOD) or 10 for limit of quantification (LOQ); $S_{y}=$ standard deviation of the $y$-intercept; and $m=$ the slope of the curve. The mass of each metal eluting from the column (as $\mathrm{pg} \mathrm{s}^{-1}$ ) was determined by dividing each baseline-corrected $\mathrm{m} / \mathrm{z}$ data point by $m$. Limits of analysis are reported in Fig. 1.

\section{Results and discussion}

Individual chromatograms for iron, copper, zinc and phosphorus are shown in Fig. 2, with the total metal per $\mathrm{mg}$ of 

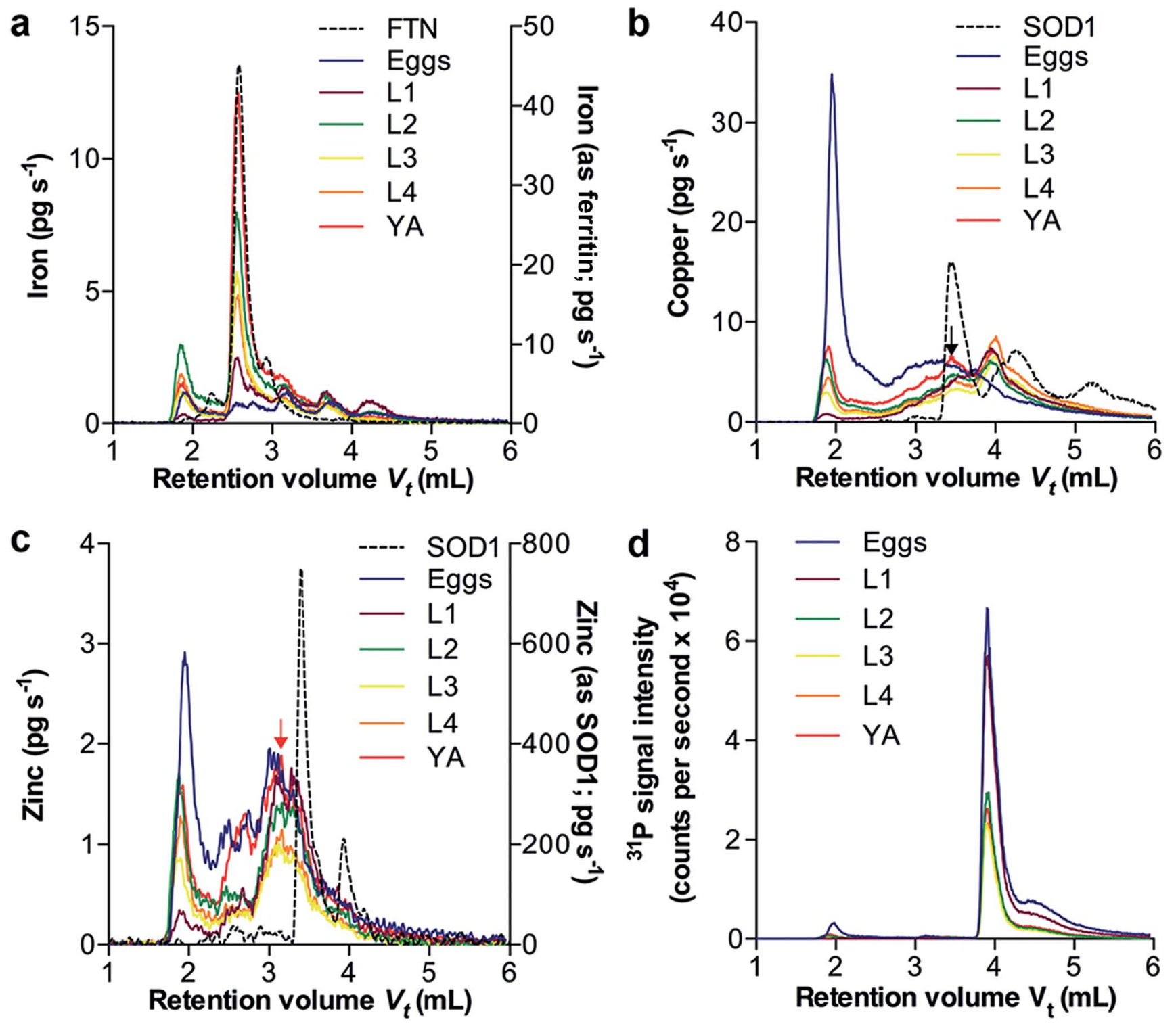

Fig. 2 Metalloproteomic and inorganic changes during C. elegans development. (a-c) Quantitative SEC-ICP-MS chromatograms show the varying concentration of metals in high, mid and low molecular-weight peaks, as well as the appearance of unique peaks during specific developmental stages. For iron (a), inclusion of a ferritin standard (right $y$-axis) shows the specific variation in iron levels in this storage protein. In the copper trace (b), the black arrow putatively denotes copper-binding Cu/Zn SOD. The red arrow in the zinc trace (c) represents a distinct peak potentially corresponding to the cytoplasmic POS-1 zinc finger protein. (d) The phosphorus chromatogram (as raw signal intensity) shows a dramatic reduction in low molecular weight phosphorus-binding species during the transition from L1 to L2.

soluble protein plotted as a function of developmental stage shown in Fig. 3.

The predominant iron-containing peak (Fig. 2a) during development at $V_{\mathrm{t}}=2.6$ was determined to be the primary $C$. elegans iron-storage protein ferritin (predominantly encoded by ftn-2 (ref. 8)) according to retention time matching with our ferritin standard. Iron bound to ferritin was near-absent in eggs, but reached its highest levels during maturation to young adult. Iron-bound ferritin did not show a linear or stage-wise increase to young adult levels; L2 specimens also showed high levels of iron associated with ferritin, as well as the most marked proportion of high-molecular weight species $\left(V_{\mathrm{t}}=1.9\right)$ compared to all other stages. Evidence of either unbound (likely as ferrous species) or low molecular weight iron-binding ligands (such as citrate and ATP) outside the permeation limit $\left(V_{\mathrm{t}}>4.0\right)$ was primarily confined to L1 larvae. The marked variation in total iron levels per mg of protein shown in Fig. 3 is most likely attributed to the high iron-binding capacity of ferritin, which can hold up to 4500 individual iron atoms. ${ }^{16}$ It is unclear as to why iron-bound ferritin levels changes, though it is likely indicative of ferritin protein expression. Ferritin expression during embryonic development is suppressed by HLH-29, ${ }^{17}$ a protein that regulates $C$. elegans ovulation ${ }^{\mathbf{1 8}}$ and may explain the limited ferritin in soluble extracts from eggs.

Copper traces showed three major peaks across each life stage $\left(V_{\mathrm{t}}=1.9 ; 3.5\right.$ and $\left.>4.0\right)$. The high molecular weight peak 


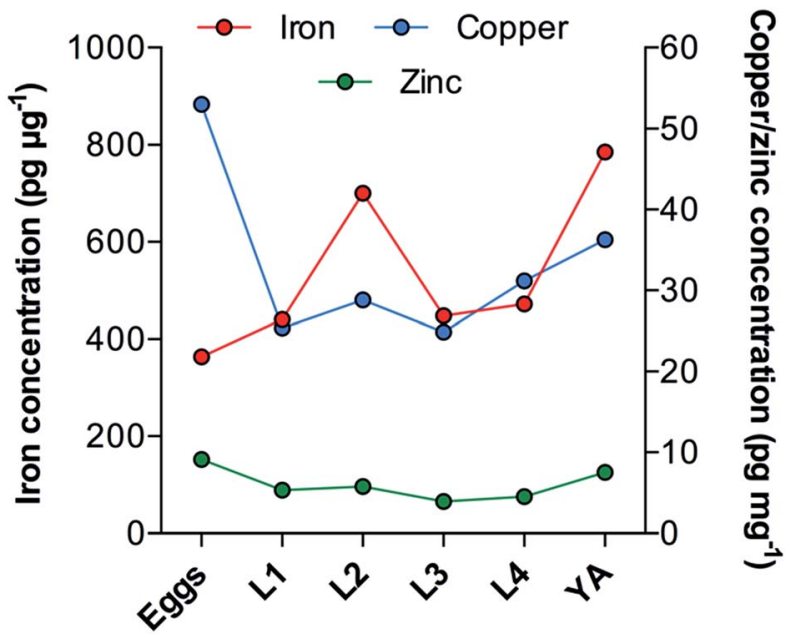

Fig. 3 Developmental changes in total metal concentration (as pg $\mu \mathrm{g}^{-1}$ ) in soluble proteins in C. elegans. Iron and copper showed a diverse pattern of changing concentration across each measured developmental stage, with zinc remaining relatively static. Specific concentrations are given in ESI Table $1 . \dagger$

was most marked in eggs and was near-absent in the following L1 stage. The peak at $V_{\mathrm{t}}=3.5$ (most prominent in the young adults) may represent $C$. elegans $\mathrm{Cu} / \mathrm{Zn}$-superoxide dismutase (SOD; encoded by the genes sod-1 and sod-5), which co-elutes with the $32 \mathrm{kDa}$ SOD1 homodimer standard, as indicated in Fig. $2 \mathrm{~b}$ by the black dotted line. In addition, larvae and young adults show a low molecular mass copper-binding species that is less pronounced in eggs.

Zinc showed a similar trend (Fig. 2c) in high molecular weight species to copper, with high levels observed in eggs, a substantial drop following hatching, and then gradual increase throughout maturation to young adult. An unknown peak at $V_{\mathrm{t}}=3.1$ was observed in all developmental stages, in addition to a peak unique to eggs and young adults at approximately $V_{\mathrm{t}}=2.7$. Soluble zinc proteins showed an approximately one order of magnitude lower concentration than cuproproteins, and the shoulder from $V_{\mathrm{t}}=3.1$ precluded alignment of any potential peak corresponding to SOD1, as seen in the copper trace. Total soluble zinc levels were also relatively stable throughout the developmental process (Fig. 3; ESI Table $1 \dagger$ ).

Comparing the proportional concentrations of iron, copper and zinc to our previously reported values in individual C. elegans,${ }^{19}$ it is apparent that the majority of zinc within C. elegans is bound to insoluble and membrane-bound proteins not detectable using our method, which is optimised to maintain native metal-ligand bonds. X-ray microfluorescence tomography showed high concentrations of zinc within nuclei ${ }^{4}$ and expression of zinc finger proteins OMA-1 and OMA-2 is enriched in this organelle. ${ }^{20}$ However, the soluble cytoplasmic zinc finger protein POS-1 (molecular mass $=55 \mathrm{kDa}$ ) is critical during early cell division, ${ }^{21}$ and may correspond to the unknown peak at $V_{\mathrm{t}}=\sim 3.1$, which exhibited decreasing amounts across larval stages.

The dramatic decrease in low molecular weight phosphorus ligands from hatching to maturation to young adults is most likely due to changing metabolic needs. Phosphorus nuclear magnetic resonance has previously shown a significant change in phosphorus-containing adenosine triphosphate (ATP), adenosine diphosphate (ADP), adenosine monophosphate (AMP) and sugar phosphates in C. elegans larval stages, ${ }^{22}$ all of which have a major effect on protein synthesis and regulation. Johnson and $\mathrm{Hirsh}^{23}$ specifically noted a major change in soluble protein expression from L1 to L2-4 stages. Therefore, the observed changes in phosphorus trace may be tied to the developing $C$. elegans metabolome. The high molecular weight species that appeared predominantly in eggs is probably yolk-lipoprotein complexes, ${ }^{24}$ consistent with embryonic metabolism.

In early development, our understanding of metalloproteomic changes at the global level is not well understood. Model systems such as C. elegans have contributed significant understanding of gene-regulatory pathways that determine key processed in development. Many of these processes are catalysed by a metal cofactor, and thus monitoring how metal levels and the proteins to which they are associated is an important step in better understanding early-life development. Specific challenges include the mapping chromatographic peaks to specific metalloproteins. For native SEC, such as the technique use here, low peak capacity can exacerbate this issue, with multiple highly relevant metalloproteins coeluting. However, few alternative techniques are available that ensure relatively weak metalprotein bonds are retained during analysis. The findings presented here represent a first step that must be improved upon if more insightful interpretations of how specific metalloprotein levels are altered during early-life. Regardless, it is consistent with metalloprotein levels existing in a dynamic state throughout development, and also provides utility in identifying specific molecular weight ligands for further proteomic analysis as part of a more comprehensive analytical approach to metalloproteomics. ${ }^{\mathbf{1 , 2 , 1 0}}$ This may include use of subsequent orthogonal chromatography of collected mass fractions, protein identification via mass fingerprinting and use of genetic manipulation to ablate candidate metalloproteins. This latter point is particularly relevant for model system approaches using yeast, C. elegans and Drosophila melanogaster.

\section{Conclusions}

Relatively little is known about regulated changes in biometal abundance and metallation status of metalloproteins during development. This proof-of-concept study demonstrates that SEC-ICP-MS can be used to examine the molecular weight distribution of metalloproteins, allow preliminary identification of proteins through retention time matching, and also identify unknown peaks for later fractionation and proteomic analysis. ${ }^{1}$ We found that the soluble metal-binding protein component of developing C. elegans is dependent on life stage, and we were able to speculate on the biological significance of these changes based on available literature. Future investigations will focus on specifically identifying the metalloproteins that exhibit changing expression over the course of C. elegans development. 


\section{Acknowledgements}

All authors are supported by an Australian Research Council Linkage Project (LP140100095) in partnership with Agilent Technologies. The Caenorhabditis Genetics Center (CGC), supported by the US National Institutes of Health-Office of Research Infrastructure Programs (P40 OD010440), provided the strain used in this study. The Victorian Government's Operational Infrastructure Support Program also supported this research.

\section{Notes and references}

1 A. Lothian, D. J. Hare, R. Grimm, T. M. Ryan, C. L. Masters and B. R. Roberts, Front. Aging Neurosci., 2013, 5, 35.

2 A. Cvetkovic, A. L. Menon, M. P. Thorgersen, J. W. Scott, F. L. Poole II, F. E. Jenney Jr, W. A. Lancaster, J. L. Praissman, S. Shanmukh, B. J. Vaccaro, S. A. Trauger, E. Kalisiak, J. V. Apon, G. Siuzdak, S. M. Yannone, J. A. Tainer and M. W. W. Adams, Nature, 2010, 466, 779-782.

3 W. A. Boyd, R. D. Cole, G. L. Anderson and P. L. Williams, Environ. Toxicol. Chem., 2003, 22, 3049-3055.

4 G. McColl, S. A. James, S. Mayo, D. L. Howard, G. F. Moorhead, D. Paterson, M. D. de Jonge and A. I. Bush, PLoS One, 2012, 7, e32685.

5 S. A. James, D. J. Hare, N. L. Jenkins, M. D. de Jonge, A. I. Bush and G. McColl, Sci. Rep., 2016, 6, 20350.

6 D. D. Shaye and I. Greenwald, PLoS One, 2011, 6, e20085.

7 B. Alberts, A. Johnson, J. Lewis, M. Raff, K. Roberts and P. Walter, Molecular Biology of the Cell, Garland Science, New York, 4 edn, 2002.

8 S. A. James, B. R. Roberts, D. J. Hare, M. D. de Jonge, I. E. Birchall, N. L. Jenkins, R. A. Cherny, A. I. Bush and G. McColl, Chem. Sci., 2015, 6, 2952-2962.
9 S. M. Yannone, S. Hartung, A. L. Menon, M. W. Adams and J. A. Tainer, Curr. Opin. Biotechnol., 2012, 23, 89-95.

10 A. Lothian and B. R. Roberts, J. Visualized Exp., 2016, e53737, DOI: $10.3791 / 53737$.

11 D. J. Hare, J. D. Doecke, N. G. Faux, A. Rembach, I. Volitakis, C. J. Fowler, R. Grimm, P. A. Doble, R. A. Cherny, C. L. Masters, A. I. Bush and B. R. Roberts, ACS Chem. Neurosci., 2015, 6, 398-402.

12 D. J. Hare, A. Grubman, T. M. Ryan, A. Lothian, J. R. Liddell, R. Grimm, T. Matsuda, P. A. Doble, R. A. Cherny, A. I. Bush, A. R. White, C. L. Masters and B. R. Roberts, Metallomics, 2013, 5, 1656-1662.

13 S. Brenner, Genetics, 1974, 77, 71-94.

14 L. Bianchi and M. Driscoll, WormBook, 2006, 1-15, DOI: 10.1895/wormbook.1.122.1.

15 T. J. Fabian and T. E. Johnson, J. Gerontol., 1994, 49, B145B156.

16 E. C. Theil, Annu. Rev. Biochem., 1987, 56, 289-315.

17 C. P. Anderson and E. A. Leibold, Front. Pharmacol., 2014, 5, 421.

18 A. White, A. Fearon and C. M. Johnson, Biol. Open, 2012, 1, 261-268.

19 K. Ganio, S. A. James, D. J. Hare, B. R. Roberts and G. McColl, Analyst, 2016, 141, 1434-1439.

20 M. R. Detwiler, M. Reuben, X. Li, E. Rogers and R. Lin, Dev. Cell, 2001, 1, 187-199.

21 B. M. Farley, J. M. Pagano and S. P. Ryder, RNA, 2008, 14, 2685-2697.

22 W. G. Wadsworth and D. L. Riddle, Dev. Biol., 1989, 132, 167173.

23 K. Johnson and D. Hirsh, Dev. Biol., 1979, 70, 241-248.

24 W. J. Sharrock, M. E. Sutherlin, K. Leske, T. K. Cheng and T. Y. Kim, J. Biol. Chem., 1990, 265, 14422-14431. 